\title{
High Speed Heterostructure Metal-Semiconductor-Metal Photodetectors
}

\author{
A. Cola ${ }^{a, *}$, B. $\mathrm{NABeT}^{b}, \mathrm{XIYing} \mathrm{Chen}^{b}$ And F. Quaranta ${ }^{a}$ \\ ${ }^{a}$ CNR-IMM sez. di Lecce, via Arnesano 73100 Lecce, Italy \\ ${ }^{b}$ Electrical and Computer Engineering Dept., Drexel University \\ 318 Commonwealth, Philadelphia, PA, 19104, USA
}

In this work we review the properties of a class of metal-semiconductormetal photodetectors based on heterojunction structures. Particularly, an $\mathrm{AlGaAs} / \mathrm{GaAs}$ device is detailed in which the absorption region is in the GaAs layer, and a two-dimensional electron gas is formed at the heterointerface due to $\delta$-doping of the widegap material. This heterostructure metalsemiconductor-metal photodetector also contains an AlGaAs distributed Bragg reflector that forms a resonant cavity for detection at $850 \mathrm{~nm}$. The beneficial effect of the two-dimensional electron gas in the GaAs absorption layer in terms of speed and sensitivity is demonstrated by comparing samples with and without doping in the AlGaAs layer. The design and the physical properties of the grown epitaxial structure are presented, together with the static and dynamic characteristics of the device in time domain. In particular, photocurrent spectra exhibit a $30 \mathrm{~nm}$ wide peak at $850 \mathrm{~nm}$, and time response measurements give a bandwidth over $30 \mathrm{GHz}$. A combination of very low dark current and capacitance, fast response, wavelength selectivity, and compatibility with high electron mobility transistors makes this device suitable for a number of application areas, such as Gigabit and 10 Gigabit Ethernet, wavelength division multiplexing, remote sensing, and medical applications.

PACS numbers: 85.60.-q, 73.40.Sx, 06.60.Jn

\section{Introduction}

As one of the most cost effective photodiodes operating at $850 \mathrm{~nm}$ wavelength for short haul, fiber optic, and free space communication, the high performance GaAs metal-semiconductor-metal (MSM) photodetectors have attracted much attention due to their planar structures, which can be easily integrated with other optoelectronic circuits and are compatible with the field effect transistors (FETs)

*corresponding author; e-mail: adriano.cola@imm.cnr.it 
process [1-3]. The novel photodetectors (PDs) for advanced Gigabit-speed fiber optic communication and fiber optics in $\mathrm{RF} /$ microwave systems are required to have fast speed, large responsivity, high signal-to-noise ratio, and wavelength selectivity. Resonant cavity enhanced devices offer the possibility of meeting some of these demands $[4,5]$. The presence of a resonant cavity creates wavelength selectivity accompanied by a drastic increase of the optical field at the resonant wavelength while the electrical properties of the photodetector remain primarily unchanged. The enhanced optical field allows photodetector to maintain high quantum efficiency as well as high speed with a thinner absorption region, while at the same time, offering narrow spectral bandwidth detection useful in wavelength-division multiplexing (WDM) applications [5]. This advantage has been utilized in the design of vertical photodetectors, such as $p-i-n$ heterojunction photodiodes, Schottky barrier internal emission photodiodes, and quantum well infrared photodetectors [6-8]. On the other hand, the FET technology itself is strongly affected by progress in heterojunction-based devices that take advantage of reduced dimensionality regime of conduction; the high electron mobility transistor (HEMT) being a prime example [9]. This has motivated the development of heterojunction-based MSM (HMSM) photodetectors that enjoy better conduction while being compatible with the HEMT technology [1,9]. With regard to this, one of the authors (BN) has recently proposed [10] a hetero-dimensional PD-based on AlGaAs/GaAs junction that shows much less dark current than conventional MSM due to the interaction between the external (3D) metal contact and the two-dimensional electron gas (2DEG) at the AlGaAs/GaAs interface. The GaAs-based HMSM photodetector with resonant cavity has been demonstrated as a promising candidate for the high performance fiber optic application, with a possibility to balance this conflict between high speed and sensitivity in the vertical direction [11, 12].

In order to further enhance the performance of an RCE-HMSM photodetector, other techniques have to be investigated. Schottky barrier height and band-edge discontinuities play an important role in the behavior of heterojunction devices $[10,13,14]$. The modulation doping technique in the heterostructure devices has been employed to increase the barrier height due to energy quantization of confined electrons [10] and through the electron-electron cloud effect [15]. The electron cloud that is formed in the narrow gap material of a heterojunction exerts a repulsive force on electrons that are emitted from the metal to the wide gap material, thus decreasing the dark current. It also influences absorption of the optically generated carriers through a field-induced index of refraction, changing the absorption coefficient similar to the Franz-Keldysh effect [16]. The most significant advantage from the modulation doping technique is that the introduction of a $\delta$-doping layer changes the two-dimensional electric field profiles from horizontally oriented to vertically oriented ones due to the screening effect of the highly crowded 2DEG formed along the interface of heterojunction, which reduces the carrier travel distance hence the transit time of carriers in the devices. It might 
be a possible solution to solve the trade-off between high speed and sensitivity in the planar direction of MSM phototectors. With the enhanced optical field by the resonant cavity and the orientation-rotated electric field by the $\delta$ modulation doping layer, high performance photodetectors are expected to be achieved without further scaling down of the devices.

Along this direction, we have recently presented a novel GaAs-based planar photodetector, combining resonant cavity, heterostructure, and $\delta$ modulation doping techniques to meet high speed, high signal-to-noise ratio, and wavelength selectivity requirements [12].

In this paper we review the characteristics of these devices, first addressing the design strategy for a GaAs-based $\delta$-doped RCE-HMSM photodetector structure. Characterizations of the grown structure are shown, which have been performed by mean of photoreflectance, photoluminescence measurements and transmission electron microscopy (TEM) observations. Then, we report a comprehensive study of the static and dynamic electrical behavior of these devices under bias. Current-voltage measurements confirm the 2DEG role in aiding photocarriers transport and in reducing dark current due to the barrier enhancement, while photocurrent measurements show optical response around $850 \mathrm{~nm}$. High speed time response measurements are given indicating that a higher peak amplitude of the transient response is achieved in presence of higher 2DEG density without degradation of temporal behavior.

\section{Device design and structure}

A typical RCE photodetector is made using a Fabry-Perot cavity, with a mirror on each end, whose length determines the resonant frequency. In practice, the bottom mirror consists of quarter-wave stacks of two different materials forming a distributed Bragg reflector (DBR). The top mirror can be the interface between the native semiconductor and air due to their large difference of refractive index; here we use a $\delta$-doped heterojunction to achieve better photon reflection as well as other important electronic functionalities. Recirculation of photons from the top of the non-absorbing barrier enhancement layer $L_{1}$ and the bottom DBR, allows a thin absorption layer $L_{2}$ to be used to minimize the response time without decreasing the quantum efficiency. Quantum efficiency, defined as the ratio of absorbed to incident photons, can be maximized when the condition $\cos \left(2\left(\beta_{1} L_{1}+\beta_{2} L_{2}\right)+\Psi_{1}+\Psi_{2}\right)=1$ is satisfied [5], where $\Psi_{1}$ and $\Psi_{2}$ are phase shifts introduced by the top and bottom mirrors, and $\beta_{1}$ and $\beta_{2}$ are the propagation constants in the two materials. There are three requirements for the materials to be selected to construct the bottom mirror: large refractive index difference, lattice matching to GaAs substrates, and bandgap larger than that of the active layer so that photons are not absorbed. For the DBR, the thickness of each individual layer in the stacks is one quarter of the effective wavelength of the incident light, which can be expressed as $\left(\lambda_{0} / n\right)$, where $\lambda_{0}$ is the incident light wavelength in a 
vacuum and $n$ is the refractive index of the material. The thickness of the spacer layer in the wide-gap material is selected to maximize the electron channel density in the narrow bandgap material but simultaneously must separate the electrons from the ionized donors at the wide bandgap material.

Based on the above considerations, the RCE heterojunction structure sketched in Fig. 1 has been realized. The layer sequence was grown by solid-source molecular beam epitaxy on a semi-insulating GaAs substrate. Twenty periods $\mathrm{Al}_{0.24} \mathrm{Ga}_{0.76} \mathrm{As} / \mathrm{Al}_{0.9} \mathrm{Ga}_{0.1} \mathrm{As} \mathrm{DBR}$ were grown on a $200 \mathrm{~nm}$ GaAs buffer layer. The thickness of each layer $\mathrm{Al}_{0.24} \mathrm{Ga}_{0.76}$ As and $\mathrm{Al}_{0.9} \mathrm{Ga}_{0.1}$ As are 596 and $676 \AA$, respectively. The bottom mirror was designed for high reflectance at $850 \mathrm{~nm}$ center wavelength. The thickness of the top barrier enhancement layer is $50 \mathrm{~nm}$ and the spacer layer is $5 \mathrm{~nm}$. A silicon $\delta$-doped layer with sheet density of $10^{13} \mathrm{~cm}^{-2}$ was grown between barrier enhancement and spacer layers. The devices, fabricated by standard photolitography, have area $40 \times 40 \mu \mathrm{m}^{2}$ with a typical interdigital pattern with a finger width of 1 or $2 \mu \mathrm{m}$ and a distance of 2 or $4 \mu \mathrm{m}$.

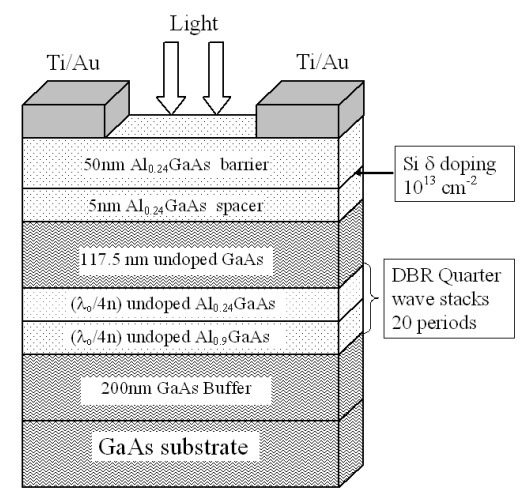

Fig. 1. Device structure of the RCE-HMSM photodetector with $\delta$-modulation doping.

For the sake of comparison, a device having the same growth structure but without Si $\delta$-doping has been fabricated. The lack of $\delta$-doping greatly affects the band bending of the underlying GaAs layer: no triangular well and 2DEG will be originated in this case.

\section{Characterization of the structure}

A relevant point in determining the performance of our photodetectors, in terms of light sensitivity, is the RCE structure which is made of a Fabry-Perot cavity. In fact, the RCE device derives advantages from the wavelength selectivity and the large increase of the resonant optical field resulting from the microcavity. The increased optical field allows photodetectors to be made thinner and therefore faster. In our case, the bottom mirror of the cavity consists of quarter-wave stacks of AlGaAs of two different compositions, forming a distributed Bragg reflector. 
The interface between the upper AlGaAs layer and air partially works as the top mirror, due to the large difference in their refractive index.

Insights of RCE structural quality can be inferred by TEM observations. Figure 2 reports the TEM micrographs of the DBR regions. The bright field image in Fig. 2a shows the alternance of the $\mathrm{Al}_{0.24} \mathrm{Ga}_{0.76} \mathrm{As}$ (measured thickness $61 \pm 1 \mathrm{~nm}$ ) and $\mathrm{Al}_{0.9} \mathrm{Ga}_{0.1}$ As layers (measured thickness $67 \pm 1 \mathrm{~nm}$ ). The quality of the interface and the inner structure of the layers can be better appreciated by the image in Fig. 2b taken at a higher resolution: absence of extended defects as well as interface abruptness are evident. The sequence of bright contrast (higher $\mathrm{Al}$ content, thickness: $3.2 \pm 0.2 \mathrm{~nm}$ ) and dark contrast (lower Al content, thickness: $1.4 \pm 0.2 \mathrm{~nm}$ ) layers is visible in $\mathrm{Al}_{0.9} \mathrm{Ga}_{0.1} \mathrm{As}$ since the average $\mathrm{Al}$ concentration of this layer was obtained by alternating the growth of thinner layers of different Al contents. The good definition of interfaces and composition of such extremely thin layer demonstrate the high structural quality of the samples.
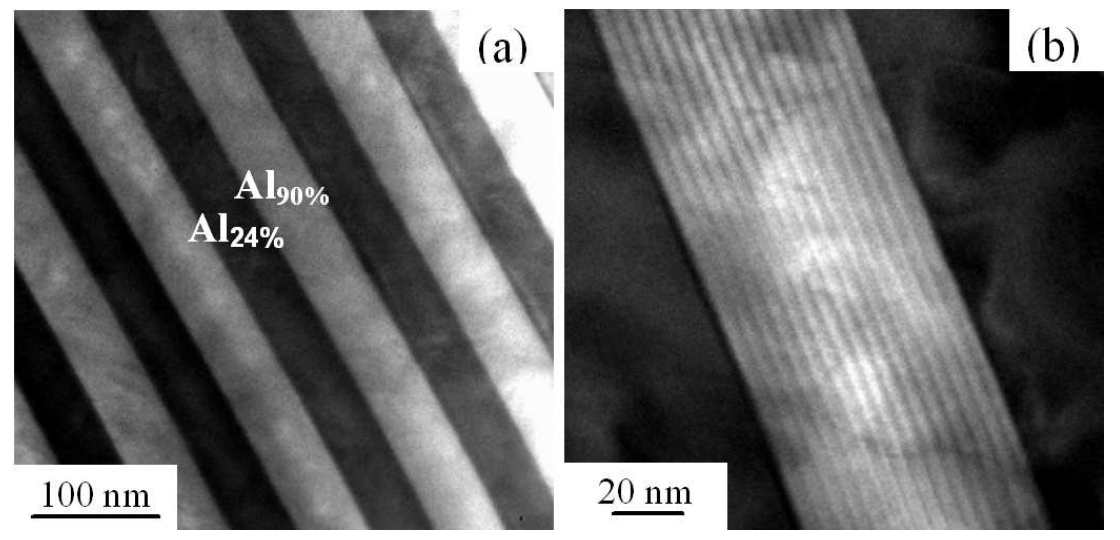

Fig. 2. (a) Low magnification TEM image of the DBR $\mathrm{Al}_{0.24} \mathrm{Ga}_{0.76} \mathrm{As} / \mathrm{Al}_{0.9} \mathrm{Ga}_{0.1} \mathrm{As}$ superlattice and (b) high magnification TEM image of the DBR showing the inner structure of the $\mathrm{Al}_{0.9} \mathrm{Ga}_{0.1}$ As layer.

In order to verify the optical spectral response of the designed structure, we have performed reflectivity measurements as a function of wavelength. The reflectivity measurement of the $\delta$-doped sample, together with a simulation curve, reported in Fig. 3, evidence that a typical optical band is well centered around the projected wavelength. Simulations, carried out by considering the designed structure sketched in Fig. 1, and assuming vertical light incidence, are in good agreement with experimental data.

Photoluminescence (PL), measured in a wavelength region around the GaAs band edge, is a suitable tool to investigate radiative transitions in the GaAs absorbing layer involving bound excitons, defects and, eventually, 2DEG at the AlGaAs/GaAs interface. Low temperature $(10 \mathrm{~K}) \mathrm{PL}$ spectra as a function of 


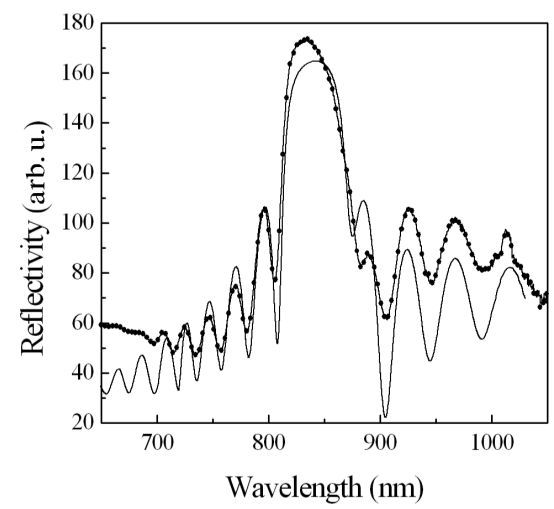

Fig. 3. Comparison of reflectivity spectrum between experimental data (dots) and simulation results (solid line) for the Si $\delta$-doped RCE-HMSM photodetector.

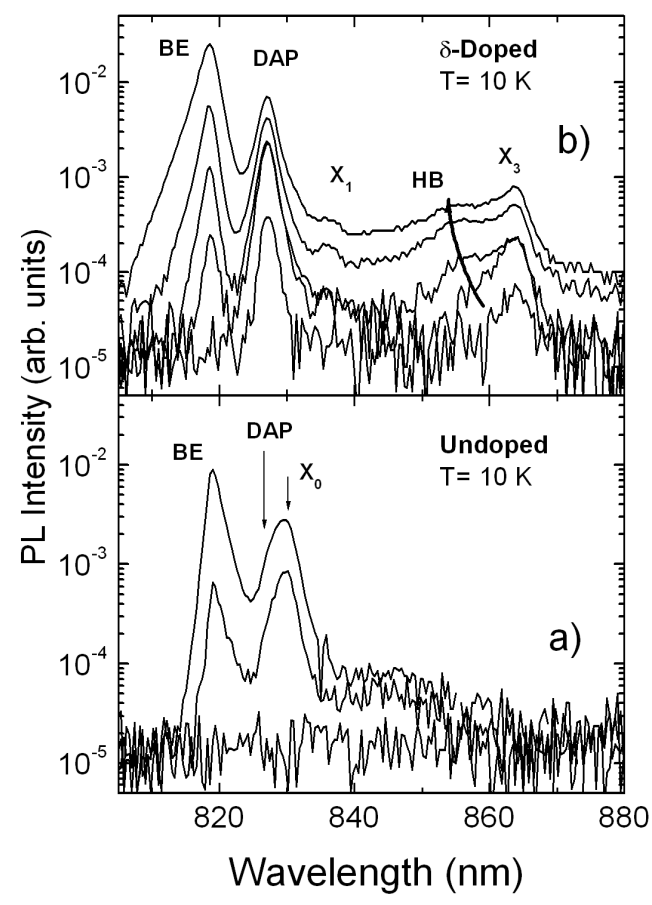

Fig. 4. Photoluminescence spectra at $10 \mathrm{~K}$ for the undoped (a) and $\delta$-doped (b) sample, for different optical powers of the excitation laser ranging between $0.016 \mathrm{~mW}$ and $2 \mathrm{~mW}$.

the optical power of the excitation source (Ar laser at $514.5 \mathrm{~nm}$ ) are shown in Fig. 4 for undoped (Fig. 4a) and $\delta$-doped (Fig. 4b) samples.

The main common features are: (i) for very low excitation power $(0.016 \mathrm{~mW})$ only the donor-acceptor pair (DAP) bands peaked at $830 \mathrm{~nm}$ are clearly visible; (ii) with increasing power density (up to $2 \mathrm{~mW}$ ) the DAP line progressively sat- 
urates in both samples and the bound exciton line BE emerges at $820 \mathrm{~nm}$ and it becomes the predominant feature for high excitation power. Additionally, in the undoped sample the DAP band is structured $\left(\mathrm{X}_{0}\right.$ line dominates about $4 \mathrm{meV}$ below the DAP emission line, see Fig. 4a), and a broad weak band appears around $850 \mathrm{~nm}$. On the other hand, the $\delta$-doped sample (Fig. 4b) presents three distinct emission bands labelled $\mathrm{X}_{1}, \mathrm{HB}$, and $\mathrm{X}_{3}$ peaked around $836 \mathrm{~nm}, 860 \mathrm{~nm}$, and $864 \mathrm{~nm}$, respectively. Line $\mathrm{X}_{3}$ is present at very low excitation power $(0.016 \mathrm{~mW})$ and it saturates at higher excitation power $(2 \mathrm{~mW})$; hence, it can be attributed to deep impurity level. On the contrary, $\mathrm{X}_{1}$ and HB emission bands emerge in the spectra with increasing excitation power and the HB line presents a clear blue-shift, of about $10 \mathrm{meV}$. We believe that these lines are related to recombinations involving the 2DEG, as reported by Bergman et al. [17]. In conclusion, PL allows us to evidence, compared to the undoped sample, the presence of the 2DEG in the $\delta$-doped sample. The photoluminescence also shows radiative transitions of bound exciton, which is indicative of the good quality of the epitaxial GaAs absorbing layer.

\section{Device characterization}

Figure 5 shows $I-V$ curves under dark and under different light excitations, for the devices with and without $\delta$-doping. Under dark, the doped sample shows a soft increase of the current with voltage, with values, at $20 \mathrm{~V}$, lower than $30 \mathrm{pA}$. The behavior of the undoped sample is rather different, as the current strongly increases at low voltage $(2-3 \mathrm{~V})$ up to about $30 \mathrm{pA}$. As the voltage is raised, the current slowly increases up to almost $100 \mathrm{pA}$ at the maximum voltage.

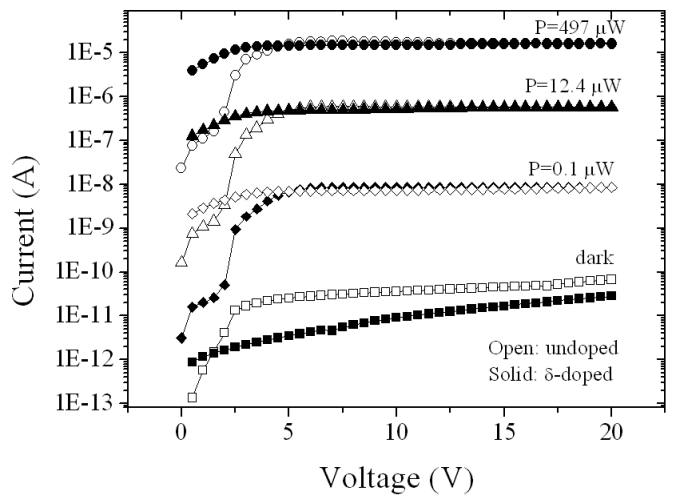

Fig. 5. Comparison of $I-V$ behavior between the $\delta$-doped (solid symbols) and undoped (empty symbols) devices under dark and light conditions. Curves corresponding to three different optical powers are reported.

It is worthwhile to note that, under dark, the lower current values of the doped sample are in qualitative agreement with the effect of the repulsion between the electrons in the metal contact and the electrons in the 2DEG; repulsion 
which is absent for the undoped samples. This effect was already observed on similar photodetectors realized with various uniform dopings of the wide-gap material [16]. The interpretation relies on Coulombic effect: as the voltage increases, the electrons of the 2DEG are pushed away from the contact and the repulsive effect decreases, thus allowing for an easier thermionic emission across the Schottky barrier. As a result, the current increases with the voltage. On the contrary, in the undoped sample there is no 2DEG below the Schottky contact and therefore, is only the blocking effect of the Schottky barrier which determines a constant current, independent of the voltage, but higher than in the previous case due to the lack of repulsive effect. In Fig. 5 there is also reported the light response under different incident optical powers of a $850 \mathrm{~nm}$ pigtailed laser, with the optical fiber of $9 \mu \mathrm{m}$ core positioned just in proximity of the active area of the device. Due to the very low leakage current and to the good responsivity, both detectors show a large dynamic response, the undoped samples showing lower photocurrent values at low voltage. However, for $V>2-3 \mathrm{~V}$, the photocurrent curves of both samples merge and strongly saturate.

The enhanced barrier height due to the $\delta$ modulation doping layer can be further evidenced in Fig. 6, where an Arrhenius plot compares dark current conduction for doped and undoped samples with a temperature range from $300 \mathrm{~K}$ to $360 \mathrm{~K}$. The activation energies can be calculated from the slopes of the $\ln I$ versus $1 / k T$ curves, which are $0.43(0.55) \mathrm{eV}$ for the undoped (doped) device under $10 \mathrm{~V}$ bias, respectively.

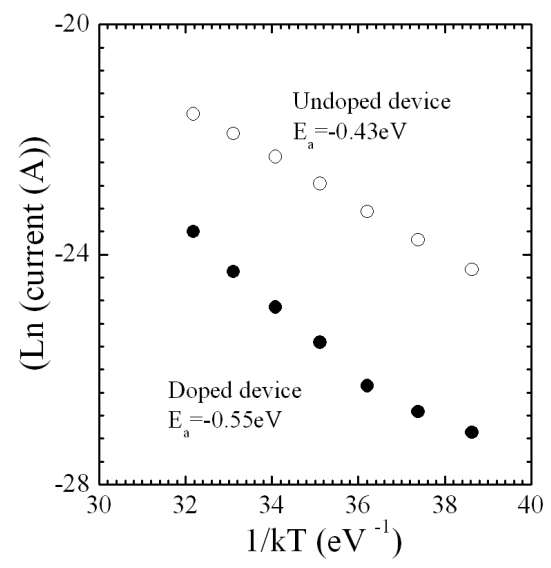

Fig. 6. Arrhenius plot for the undoped (empty circles) and $\delta$-doped (solid circles) devices at $10 \mathrm{~V}$.

By using a precision LCR meter, we performed capacitance measurements at $1 \mathrm{MHz}$ frequency and $30 \mathrm{mV}$ amplitude of the oscillating voltage, on doped and undoped devices. The measurements have been carried out paying attention to the sample shielding, minimizing cable length, and substracting the stray capacitance. 
TABLE I

Measured and calculated geometrical capacitance values of different interdigital $\delta$-doped and undoped devices.

\begin{tabular}{c|c|c|c}
\hline \hline $\begin{array}{c}\text { Interdigital structure } \\
\text { parameters } \\
\text { width }(W) \text {,gap }(G)\end{array}$ & $\begin{array}{c}\text { Experimental } \\
\delta \text {-doped } \\
C(\mathrm{fF})\end{array}$ & $\begin{array}{c}\text { Experimental } \\
\text { undoped } \\
C(\mathrm{fF})\end{array}$ & $\begin{array}{c}\text { Calculated } \\
\text { values } \\
C(\mathrm{fF})\end{array}$ \\
\hline$W=1 \mu \mathrm{m}, G=2 \mu \mathrm{m}$ & 24.4 & 26 & 27 \\
$W=1 \mu \mathrm{m}, G=4 \mu \mathrm{m}$ & 11.5 & 16.5 & 13 \\
$W=2 \mu \mathrm{m}, G=2 \mu \mathrm{m}$ & 21.0 & 21.5 & 26.5 \\
$W=2 \mu \mathrm{m}, G=4 \mu \mathrm{m}$ & 14.4 & 16 & 14
\end{tabular}

We observe in Table I the good agreement between the capacitance values, as measured at high voltages, and the geometrical values, calculated from the proper relation [18].

Figure 7 illustrates the experimental photocurrent spectral response of the doped and undoped RCE-HMSM photodetectors. A monochromator with $0.15 \mathrm{~nm}$ resolution was used to select the excitation wavelength from a chopped tungsten light source. The signal was measured by a lock-in amplifier and the spectral response was measured under $10 \mathrm{~V}$ reverse bias. The resonant peak is around $850 \mathrm{~nm}$ and the FWHM value is $30 \mathrm{~nm}$. An increase of photocurrent is observed

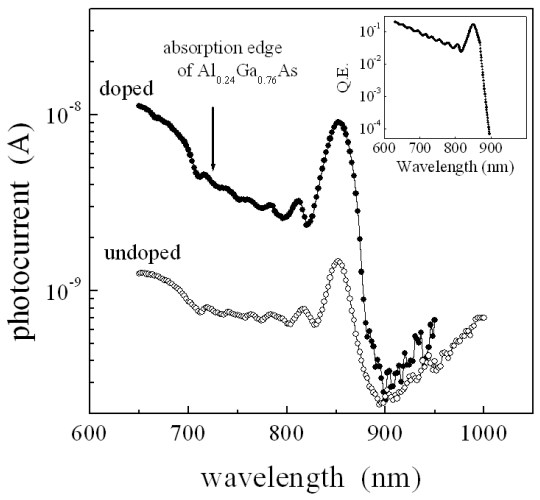

Fig. 7. Photocurrent spectral response for the undoped device (empty circles) and the $\delta$-doped (solid circles) device; the inset shows the calculated quantum efficiency.

around $710 \mathrm{~nm}$, which is due to absorption in AlGaAs layers. We note that the spectral response is, at all the wavelengths, much higher for the doped than for the undoped device. This is consistent with the photocurrent response to the monochromatic laser at $850 \mathrm{~nm}$ (Fig. 5), by taking into account that in Fig. 6 the light intensity is very low and the applied voltage is $10 \mathrm{~V}$. Some photocurrent oscillations are evident between $720 \mathrm{~nm}$ and $850 \mathrm{~nm}$ in both devices which can be 
attributed to the effect of the Bragg reflector. The inset shows the simulation of the internal quantum efficiency, defined as the ratio of absorbed to incident photons. A clear resonant peak at $850 \mathrm{~nm}$ with $26 \mathrm{~nm}$ FWHM is observed and at lower wavelengths, oscillations and a gradually increasing shoulder are observed. All these features are in good agreement with the experimental data. This indicates that the optical properties due to the layered structure are responsible for the features of the spectral response and, at the same time, the amplitude difference between the two experimental curves can only be explained in terms of transport and collection of the photogenerated carriers.

High-speed time response measurements were made using a mode-locked Ti: sapphire laser, operating with a repetition rate of $76 \mathrm{MHz}$ that generated $100 \mathrm{fs}$ pulses. The wavelength of the Ti:sapphire laser was tuned in the range of 830 $880 \mathrm{~nm}$ while the optical power was maintained at $0.1 \mathrm{~mW}$ by a suitable filter, in order to verify the wavelength selectivity under dynamic operations.

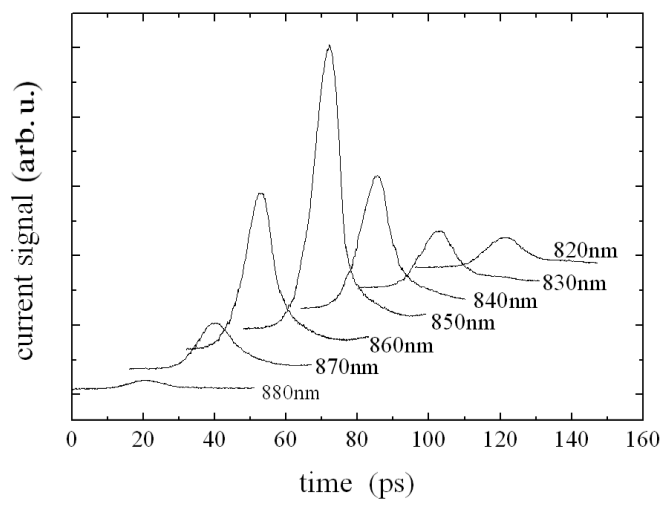

Fig. 8. Temporal response of an undoped device at different wavelengths of impinging pulses.

Figure 8 shows the temporal response of a $\delta$-doped photodetector, with a $1 \mu \mathrm{m}$ finger and $4 \mu \mathrm{m}$ spacing between fingers, measured by a $50 \mathrm{GHz}$ sampling scope at $20 \mathrm{~V}$ bias, for various wavelengths. We notice that time traces show the maximum sensitivity around $850 \mathrm{~nm}$, consistently with dc photocurrent results.

We have then set the laser wavelength at $850 \mathrm{~nm}$ and performed systematic measurements on the devices of different contact geometries, comparing $\delta$-doped and undoped devices, biased at $20 \mathrm{~V}$. Table II, which summarizes the results, reports the peak amplitude, the peak FWHM, and the frequency cut-off. The peak amplitude, which has been properly normalized to take into account the active area of the device, is always greater for the doped samples. This result confirms the photocurrent measurements carried out under static conditions. The samples with $G=2 \mu \mathrm{m}$ have a peak amplitude always greater than those with $G=4 \mu \mathrm{m}$, for each $W$. This is indicative of incomplete collection of charge, 
probably due to trapping processes. The pulse widths of the undoped samples of different $W$ and $G$ are comparable among them; the same holds for the doped samples (except one). However, the doped samples have pulse width shorter than undoped samples indicating a higher detector bandwidth. The cut-off frequencies calculated from the fast Fourier transform of the temporal response, also reported in Table II, show the values for $\delta$-doped devices in the range of $25-44 \mathrm{GHz}$, and the values for the undoped device in the range of $24-26 \mathrm{GHz}$. These results are in agreement with the aiding role of the internal vertical field introduced by the 2DEG [16] and suggest that high performance devices can be designed insensitive to the finger geometry.

TABLE II

Pulse amplitude, width, and cut-off frequency from time response of different interdigital $\delta$-doped and undoped devices, biased at $20 \mathrm{~V}$.

\begin{tabular}{c|c|c|c|c|c|c}
\hline \hline $\begin{array}{c}\text { Interdigital structure } \\
\text { parameters } \\
\text { width }(W) \text {, gap }(G)\end{array}$ & $\begin{array}{c}\text { Amplit. } \\
\delta \text {-doped } \\
\text { (arb.u.) }\end{array}$ & $\begin{array}{c}\text { Amplit. } \\
\text { undoped } \\
\text { (arb.u.) }\end{array}$ & $\begin{array}{c}\text { Width } \\
\delta \text {-doped } \\
(\mathrm{ps})\end{array}$ & $\begin{array}{c}\text { Width } \\
\text { undoped } \\
(\mathrm{ps})\end{array}$ & $\begin{array}{c}\text { Cut-off } \\
\text { freq. } \\
\text {-doped } \\
(\mathrm{GHz})\end{array}$ & $\begin{array}{c}\text { Cut-off } \\
\text { freq. } \\
\text { undoped } \\
(\mathrm{GHz})\end{array}$ \\
\hline$W=1 \mu \mathrm{m}, G=2 \mu \mathrm{m}$ & 0.331 & 0.192 & 7.97 & 9.45 & 44.0 & 26.9 \\
$W=1 \mu \mathrm{m}, G=4 \mu \mathrm{m}$ & 0.292 & 0.207 & 11.9 & 10.38 & 25.6 & 25.6 \\
$W=2 \mu \mathrm{m}, G=2 \mu \mathrm{m}$ & 0.252 & 0.103 & 7.03 & 10.16 & 34.2 & 29.3 \\
$W=2 \mu \mathrm{m}, G=4 \mu \mathrm{m}$ & 0.206 & 0.111 & 7.11 & 12.04 & 26.3 & 24.4
\end{tabular}

In conclusion, we propose that the increase in responsivity and response speed is attributed to the enhanced optical field with the resonant cavity structure and the orientation-rotated electric field and modified potential profile by the $\delta$ modulation doped layer, while the decrease of dark current is due to the confined electron cloud.

\section{Conclusions}

In this work we have fully characterized a novel HMSM Schottky photodetector, and we have verified the performance improvement due to the $\delta$ modulation doping. In addition, the suitable employment of the vertical resonance cavity is useful in solving the trade-off between high quantum efficiency and high speed while, at the same time, offering narrow spectral bandwidth detection.

The concurrent improvement in spectral response, in dark current, in dc photoresponse, and in time response, is attributed to the presence of the $\delta$-doped sheet in the upper AlGaAs layer, playing an important role, in terms of barrier enhancement and introducing an internal electric field that aids in transport of generated photocarriers. The $\delta$-doped devices exhibited very low dark current and capacitance, wavelength selectivity, high quantum efficiency. Combination of low 
dark current and capacitance, fast response, wavelength selectivity and integrability with high electron mobility transistors makes the $\delta$-doped device especially suitable for high sensitivity photodetection in general and for Gigabit Ethernet, 10 Gigabit Ethernet, OC 48 and OC192 optical communications applications.

\section{References}

[1] P. Fay, C. Caneau, I. Adesida, IEEE Trans. Microw. Theory Tech. 50, 62 (2002).

[2] A. Jayakumar, M.S. Bustos, D. Cheskis, S.J. Pietrucha, M. Bonelli, S. Al-Kuran, N. Scheinberg, IEEE J. Solid-State Circuits 35, 1271 (2000).

[3] M. Lang, W. Bronner, W. Benz, M. Ludwig, V. Hurm, G. Kaufel, A. Leuther, J. Rosenzweig, M. Schlechtweg, Electron. Lett. 37, 1247 (2001).

[4] A. Strittmatter, S. Kollakowski, E. Droge, E.H. Bottcher, D. Bimberg, Electron. Lett. 32, 1231 (1996).

[5] M.S. Unlu, S. Strite, J. Appl. Phys. 78, 607 (1995).

[6] I. Kimukin, N. Biyikli, B. Butun, O. Aytur, M.S. Unlu, E. Ozbay, IEEE Photonics Technol. Lett. 14, 366 (2002).

[7] I. Kimukin, E. Ozbay, N. Biyikli, T. Kartaloglu, O. Aytür, M.S. Unlu, G. Tuttle, Appl. Phys. Lett. 77, 3890 (2000).

[8] A. Shen, H.C. Liu, M. Gao, E. Dupont, M. Buchanan, J. Ehret, G.J. Brown, F. Szmulowicz, Appl. Phys. Lett. 77, 2400 (2000).

[9] J.H. Burroughes, IEEE Photonics Technol. Lett. 3, 660 (1991).

[10] B. Nabet, IEEE Photonics Technol. Lett. 9, 223 (1997).

[11] Xiying Chen, B. Nabet, F. Quaranta, A. Cola, M. Currie, Appl. Phys. Lett. 80, 3222 (2002).

[12] Xiying Chen, B. Nabet, A. Cola, F. Quaranta, M. Currie, IEEE Electron Device Lett. 24, 312 (2003).

[13] F. Capasso, Surf. Sci. 142, 513 (1984).

[14] F. Capasso, A.Y. Cho, K. Mohammed, P.W. Foy, Appl. Phys. Lett. 46, 664 (1985).

[15] A. Anwar, B. Nabet, IEEE Trans. Microw. Theory Tech. 50, 68 (2002).

[16] B. Nabet, A. Cola, F. Quaranta, M. Cesareo, R. Rossi, R. Fucci, A. Anwar, Appl. Phys. Lett. 77, 4007 (2000).

[17] J.P. Bergman, Q.X. Zhao, P.O. Holtz, B. Monemar, M. Sundaram, J.L. Merz, A.C. Gossard, Phys. Rev. B 43, 4771 (1991).

[18] J.B.D. Soole, H. Schumacher, IEEE Trans. Electron Devices 37, 2285 (1990). 